\title{
Meridià: un portal para la difusión de la ciencia desde una óptica integral y cooperativa
}

\author{
Por Llorenç Arguimbau, Aldara Cervera, Robert Latorre y Mercè Martí
}

\begin{abstract}
Resumen: El buen funcionamiento del ciclo de la investigación, el desarrollo y la innovación $(I+D+I)$ requiere un tratamiento eficaz de la información científica. El Institut d'Estudis Catalans (IEC) dispone de un observatorio científico que, recientemente, ha presentado el portal web Meridià. Desde un punto de vista global, este proyecto tiene la voluntad de ser una iniciativa cooperativa para integrar y compartir información con los otros agentes del sistema de la ciencia, facilitándoles un conocimiento exhaustivo de su entorno y de la evolución de las diferentes áreas científicas y tecnológicas.

Palabras clave: Investigación, Desarrollo, Innovación, Información científica, Observatorio, Portal Meridià.

Title: Meridià: a web portal to disseminate science from a comprehensive and cooperative view
\end{abstract}

Abstract: The proper functioning of the Research, Development and Innovation (R\&D\&I) cycle requires an efficient processing of scientific information. The Institute for Catalan Studies (IEC) possesses a scientific observatory which recently presented the web portal Meridià. From a global point of view, this project aims to be a cooperative initiative to integrate and share information with other agents of the scientific system, providing a thorough understanding of their environment and the evolution of the different areas in science and technology.

Keywords: Research, Development, Innovation, Information Science, Observatory, Meridià portal.

Arguimbau, Llorenç; Cervera, Aldara; Latorre, Robert; Martí, Mercè. "Meridià: un portal para la difusión de la ciencia desde una óptica integral y cooperativa”. El profesional de la información, 2009, septiembre-octubre, v. 18, n. 5, pp. 540-544.

DOI: $10.3145 /$ epi.2009.sep.08

\section{Introducción}

EL CICLO DE LA INVESTIGACIÓN, el desarrollo y la innovación $(\mathrm{I}+\mathrm{D}+\mathrm{I})$ ocupa una posición estratégica al potenciar el conocimiento científico y el crecimiento económico. Para el buen funcionamiento de este ciclo, es imprescindible llevar a cabo un tratamiento eficaz de la información científica.

Hacer ciencia es sinónimo de hacer públicos los resultados (Rodríguez-López, 2005). Por lo tanto, los análisis documentales sobre la ciencia se han centrado en áreas como el movimiento Open Access $(O A)$, la bibliometría o, más recientemente, la Ciencia 2.0 (Cabezas-Clavijo; Torres-Salinas; Delgado-López-Cózar, 2009; Codina, 2009). Cabe reivindicar una perspectiva más amplia que reúna los recursos, los resultados y las formas de organización involucrados en la creación de conocimiento (Callon; Courtial; Penan, 1995). Para impulsar la investigación, ésta ha de estudiarse científicamente, aplicando con rigor el mismo método científico.

A partir de 1990, con la creación en Francia del Observatoire des Sciences et des Techniques $(O S T)$, este tipo de organismos ha proliferado en diversos países. Los 
observatorios de ciencia y tecnología pueden definirse como organizaciones concebidas para diseñar, integrar y producir información, indicadores y análisis sobre el sistema nacional de I+D+I bajo los estándares y metodologías internacionales (OCDE, 2003; De-la-Vega, 2007).

La tarea de descripción y valoración de un sistema de I+D+I no resulta nada fácil (Fuentes; Arguimbau, 2008). Hay una gran atomización de los datos, derivada de la enorme cantidad de agentes ejecutores y financiadores, que se caracterizan por su pluralidad (naturaleza jurídica, sector de trabajo, dimensiones) y por su interacción dinámica (redes, depósitos de conocimiento, etc.). Además, surgen constantemente nuevas áreas, conceptos, métodos, técnicas y espacios interdisciplinares.

\section{Marco organizativo}

En los últimos años, el Institut d'Estudis Catalans (IEC) ha ejercido un papel cada vez más importante como entidad consultora de los agentes públicos, mediante la elaboración de informes y dictámenes sobre aspectos estratégicos de política científica. El IEC, la academia catalana de las ciencias y las humanidades, se creó en 1907 como un instrumento al servicio de las políticas de investigación y difusión del conocimiento. Después de un largo período de clandestinidad, un Real decreto aprobado el 26 de noviembre de 1976 le otorgaba reconocimiento oficial como "corporación académica que tiene por objeto la alta investigación científica". Su espacio de actuación se extiende a los territorios de lengua y cultura catalanas.

En 1995, por encargo de la $G e$ neralitat de Catalunya, el IEC puso en marcha el proyecto Reports de la recerca a Catalunya. Se trata de una serie de informes por materias específicas sobre el estado de la investigación en Catalunya y redacta- dos por equipos de investigadores de prestigio. Por ahora se han publicado dos ediciones, que abarcan los períodos 1990-1995 (24 informes) y 1996-2002 (27 informes).

Para facilitar un apoyo documental a las tareas de elaboración de la segunda edición de los Reports, el IEC creó en 2003 el Observatori de la Recerca de l'Institut d'Estudis Catalans (OR-IEC). Desde entonces, el OR-IEC ha ido incorporando datos detallados sobre el sistema I+D+I de las zonas de lengua y cultura catalanas, con comparativas nacionales e internacionales.

\section{EI portal Meridià}

Durante 2008 el IEC puso en marcha el portal web Meridià (Medición de la investigación, el desarrollo y la innovación) (figura 1), un servicio ideado para diferentes tipos de clientes. Entre los puntos fuertes del proyecto destaca la globalidad del público (universidades, empresas, administraciones públicas, entidades privadas sin ánimo de lucro, etc.) y de los temas (ciencias, ciencias de la vida y de la salud, ingenierías y arquitectura, ciencias sociales y humanidades). Con la finalidad de organizar y facilitar la comparación de los datos, se trabaja con la clasificación de las áreas científicas de los Reports y se presentan equivalencias con otras taxonomías estandarizadas.

El propósito básico de Meridià es impulsar y difundir la ciencia a partir de un conocimiento detallado de todo el ciclo de la investigación científica, desde los inputs recibidos hasta los outputs obtenidos: contexto socioeconómico, organismos de investigación, recursos (humanos, económicos y materiales) y, finalmente, resultados (artículos, patentes y tesis). El enfoque evolutivo del proyecto parte de la recopilación de los datos dispersos y heterogéneos, continúa con su transformación en información incorporándola a la plataforma de Meridià y acaba con el tratamiento de la información para la generación de conocimiento y la consiguiente toma de decisiones. La utilidad de la información contenida en el proyecto es muy diversa: desde respuestas a preguntas concretas (servicio de referencia virtual) hasta la elaboración de informes estratégicos.

El portal se encuentra disponible en catalán, castellano e inglés y presenta la siguiente información (tabla 1):

- Informes estadísticos y dinámicos, con datos metodológicos sobre alcance geográfico y temporal, fuente de datos, etc.

- Documentos (memorias, informes, planes de I+D+I, etc.).

- Motor de búsqueda en la base de datos y los documentos.

- Noticias de actualidad.

- Enlaces web.

\begin{tabular}{|l|r|}
\hline \multicolumn{1}{|c|}{ Categorías } & í tems \\
\hline Clasificaciones & 26 \\
\hline Entidades & 12.765 \\
\hline $\begin{array}{l}\text { Recursos económicos } \\
\text { (expedientes) }\end{array}$ & 68.500 \\
\hline Recursos humanos & 37.429 \\
\hline Resultados (revistas) & 1.092 \\
\hline $\begin{array}{l}\text { Resultados } \\
\text { (tesis doctorales) }\end{array}$ & 11.621 \\
\hline Resultados (patentes) & 8.795 \\
\hline
\end{tabular}

Tabla 1. Información de la base de datos Meridià

Meridià dispone de diversos niveles de consulta de la información, desde el privilegiado para los organismos con los que se establecen convenios, hasta la información básica para el público en general. Así, el sistema resulta flexible para establecer perfiles específicos para cada grupo de usuarios potenciales:

- Cargos políticos de las administraciones públicas. 


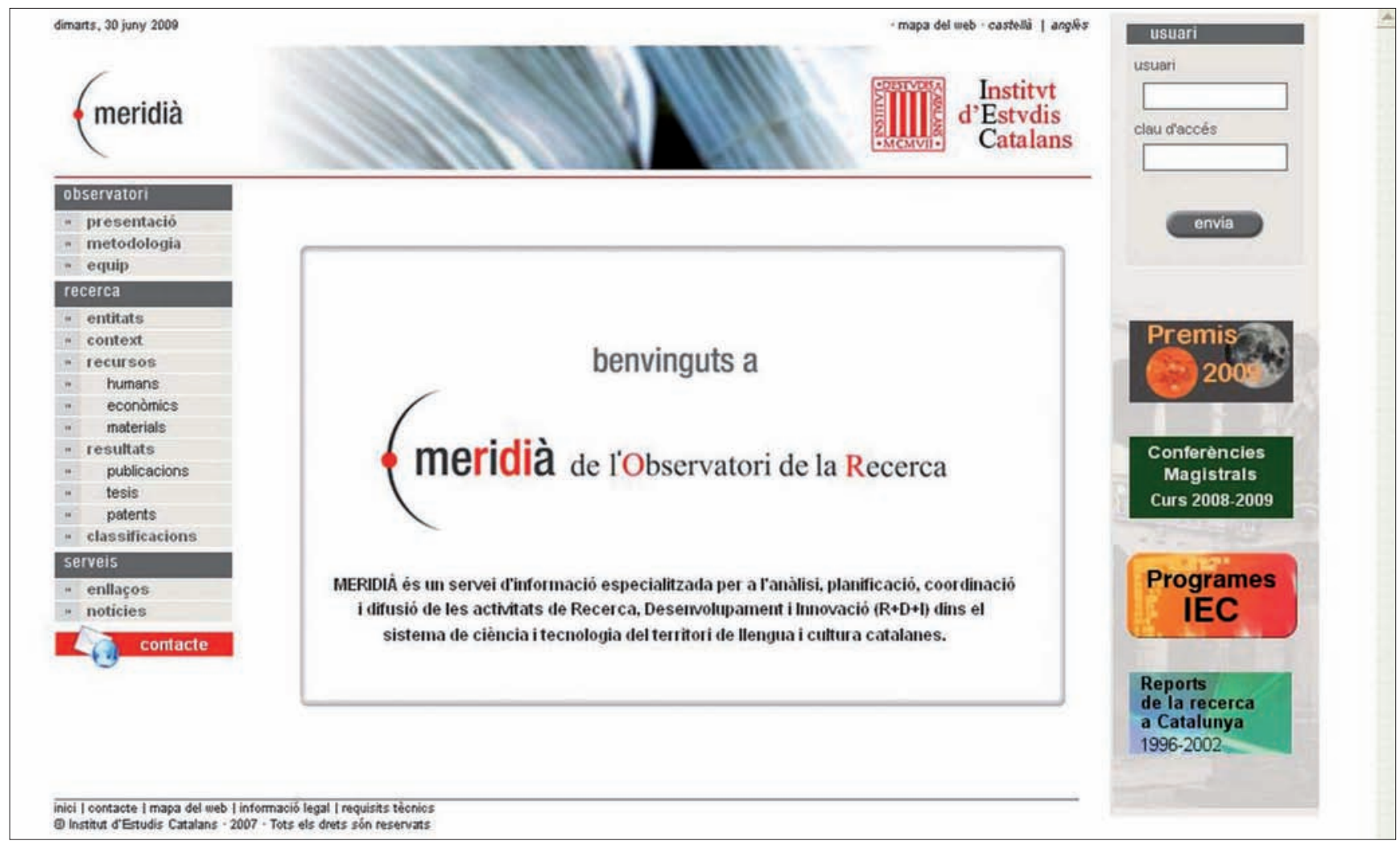

Figura 1. Meridià. http://meridia.iec.cat

- Rectores y vicerrectores de las universidades públicas y privadas.

- Directores y responsables de organismos, institutos y centros de investigación.

- Personal gestor y técnico en política científica y tecnológica.

- Miembros, personal y socios de las sociedades filiales del IEC.

- Personal investigador especializado en la descripción, el análisis y la prospectiva de las diferentes actuaciones de $\mathrm{I}+\mathrm{D}+\mathrm{I}$.

- Comunidad científica y sociedad en general.

Este proyecto es una iniciativa cooperativa para integrar y compar-

\footnotetext{
"Se ofrecen desde respuestas a preguntas concretas hasta la elaboración de informes estratégicos"
}

tir información con el resto de los agentes del sistema de I+D+I, facilitándoles un conocimiento exhaustivo de su entorno y de la evolución de las diferentes áreas científicas y tecnológicas (no se realizan análisis comparativos entre entidades ni investigadores). Todos los datos sobre recursos y resultados de que se dispone son fruto de la tarea documental llevada a cabo por el OR-IEC, que contempla la identificación, selección y vaciado sistemático de múltiples fuentes de información (figura 2) y el tratamiento informático de los datos recopilados, que provienen básicamente de dos tipos de fuentes:

- Convenios para la transferencia de información con organismos públicos y privados vinculados a la investigación.

- Procesamiento sistemático de fuentes de información primarias (boletines oficiales, registros administrativos, etc.) y secundarias (memorias, informes, etc.).

Como se puede comprobar, las fuentes son públicas y oficiales y eso determina que se pueda hablar de datos sólidos y fiables que, una vez tratados y normalizados se incorporan a una base de datos para su posterior difusión. A modo de aplicación interna, el OR-IEC también dispone de una intranet donde se conservan documentos y fuentes vinculados con la investigación (informes, memorias, resoluciones de convocatorias, sitios web de interés, etc.).

"Se trata de mejorar el conocimiento de las áreas científicas y tecnológicas de los agentes del sistema de I+D+l'

Además del portal web que muestra los resultados generados por el $O R-I E C$, el diseño tecnológico de Meridià presenta una plataforma con diferentes módulos: 


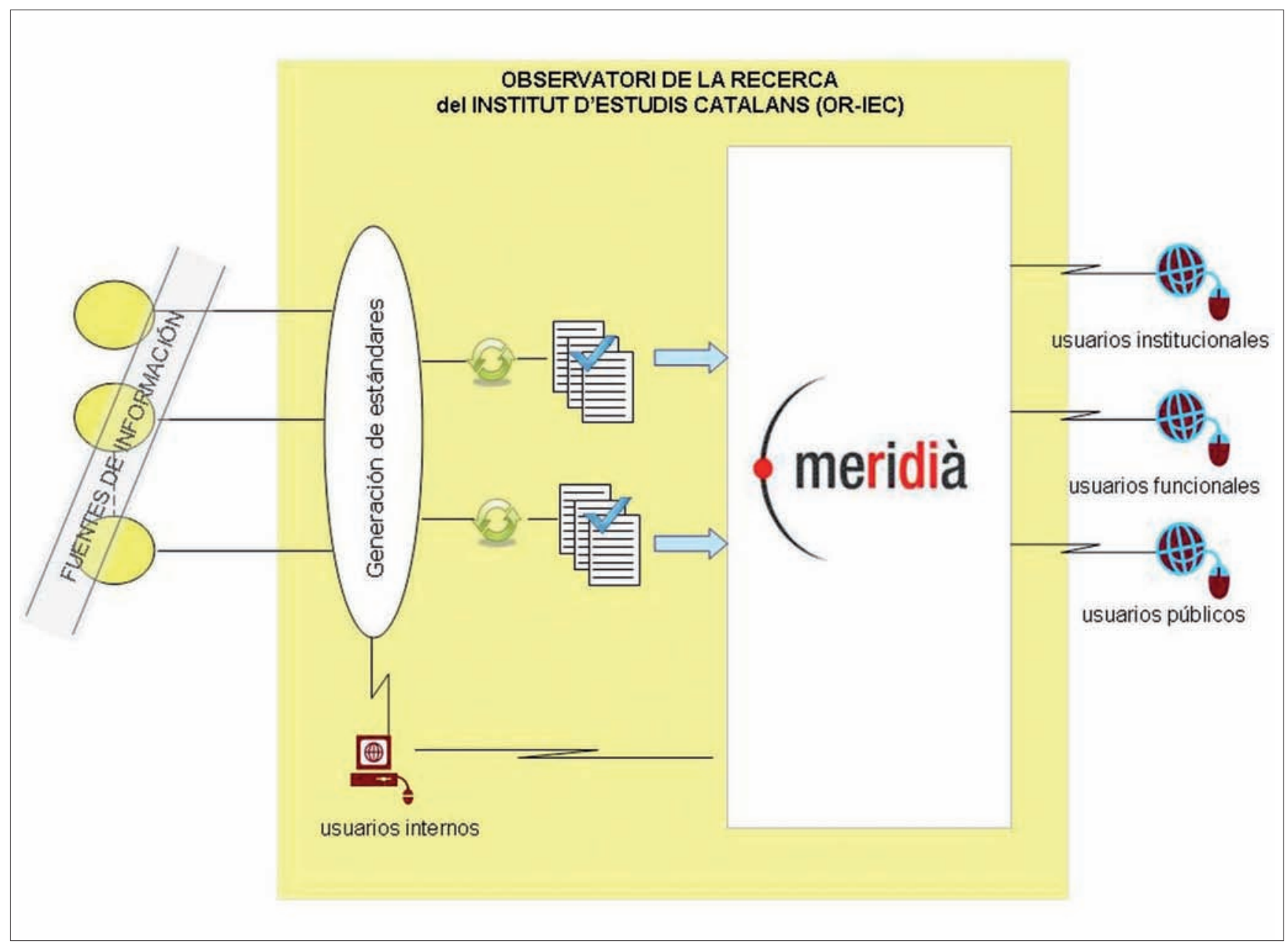

Figura 2. Meridià. Proceso de adquisición y carga de datos

- Base de datos construida sobre un esquema conceptual flexible para adaptarse a la complejidad de las múltiples fuentes de información.

- Entorno interno que facilita las tareas de administración de la información. Esta aplicación y el portal se han implementado en lenguaje Java con el Framework Struts y se han desplegado en un servidor de aplicaciones Apache Tomcat 5.5. La capa de datos utiliza la base de datos Oracle $10 \mathrm{GR}$ y ficheros xml donde se almacenan los contenidos no estructurados (noticias, enlaces, documentos, etc.).

- Entorno analítico que garantiza la explotación de los datos para generar informes. Técnicamente, se ha confeccionado con MicroStrategy 8.0 y con el componente de visualización de informes IEPortal.
A partir de la experiencia adquirida por el $O R-I E C$ y de los comentarios recibidos, se prepara la fase 2 del portal que contempla una serie de nuevos servicios y mejoras funcionales que se agrupan en las siguientes líneas de trabajo:

- Rediseñar la interfaz Meridià y orientarla hacia un usuario no experto.

- Abrir una parte sustancial de los contenidos a todo tipo de usuarios.

- Aumentar la interacción con usuarios y sistemas externos.

- Desplegar un motor de búsqueda integral.

\section{Consideraciones finales}

Meridià es un observatorio interactivo para facilitar los datos y los instrumentos de búsqueda y análisis a un público potencial. $\mathrm{Su}$ finalidad es impulsar y difundir la ciencia a partir de un conocimiento de los fondos invertidos y de los resultados obtenidos. Se trata de una iniciativa del $I E C$ con un gran potencial de crecimiento futuro a partir de la cooperación con el resto de agentes científicos y tecnológicos. Meridià aspira a ser un eslabón más en la función original de internet, es decir, potenciar la red de redes como una comunidad real para el conocimiento científico.

\section{Bibliografía}

Arguimbau, Llorenç; Cervera, Aldara; Latorre, Robert; Martí, Mercè; Guerrero, Ricard. "El portal Meridià de l'Observatori de la Recerca (OR-IEC): anàlisi, promoció i difusió del coneixement científic català". Coneixement $i$ societat, 2008 , n. 15 , pp. 80-105. http://eprints.rclis.org/15676

Cabezas-Clavijo, Álvaro; Torres-Salinas, Daniel; Delgado-López-Cózar, Emilio. "Ciencia 2.0: catálogo de herramientas e implicaciones 
para la actividad investigadora". El profesional de la información, 2009, v. 18, n. 1, pp. 72-79.

Callon, Michel; Courtial, Jean-Pierre; Penan, Hervé. Cienciometría: la medición de la actividad científica: de la bibliometría a la vigilancia tecnológica. Gijón: Trea, 1995.

Codina, Lluís. "Ciencia 2.0: redes sociales y aplicaciones en línea para académicos". Anuario hipertext.net, 2009, n. 7 .

http://www.hipertext.net/web/pag295.htm

De-la-Vega, Iván. "Tipología de observatorios de ciencia y tecnología. Los casos de América Latina y Europa". Revista española de documentación científica, 2007, v. 30, n. 4, pp. 545-552.
Fuentes-Pujol, Eulàlia; Arguimbau, Llorenç. "I+D+I: una perspectiva documental". Anales de documentación, 2008, n. 11, pp. 43-56.

http://revistas.um.es/analesdoc/article/viewFi le/24801/24121

Ocde. Manual de Frascati 2002: propuesta de norma práctica para encuestas de investigación y desarrollo experimental. Madrid: Fecyt, 2003.

Rodríguez-López, Joaquín. "Ciencia y comunicación científica: edición digital y otros fundamentos del libre acceso al conocimiento". El profesional de la información, 2005, v. 14, n. 4, pp. 246-254.

Rovira-Fernàndez, Anna. "Les biblioteques universitàries i el suport a la recerca". BiD: textos universitaris de biblioteconomia i documentació, 2007, n. 18.

http://www.ub.edu/bid/pdf/18rovir1.pdf

\section{Llorenç Arguimbau, Aldara Cer-} vera, Robert Latorre, Mercè Martí Institut d'Estudis Catalans. C/Carme, 47, 08001 Barcelona.

llarguimbau@iec.cat

acervera@iec.cat

rlatorre@iec.cat

mmarti@iec.cat

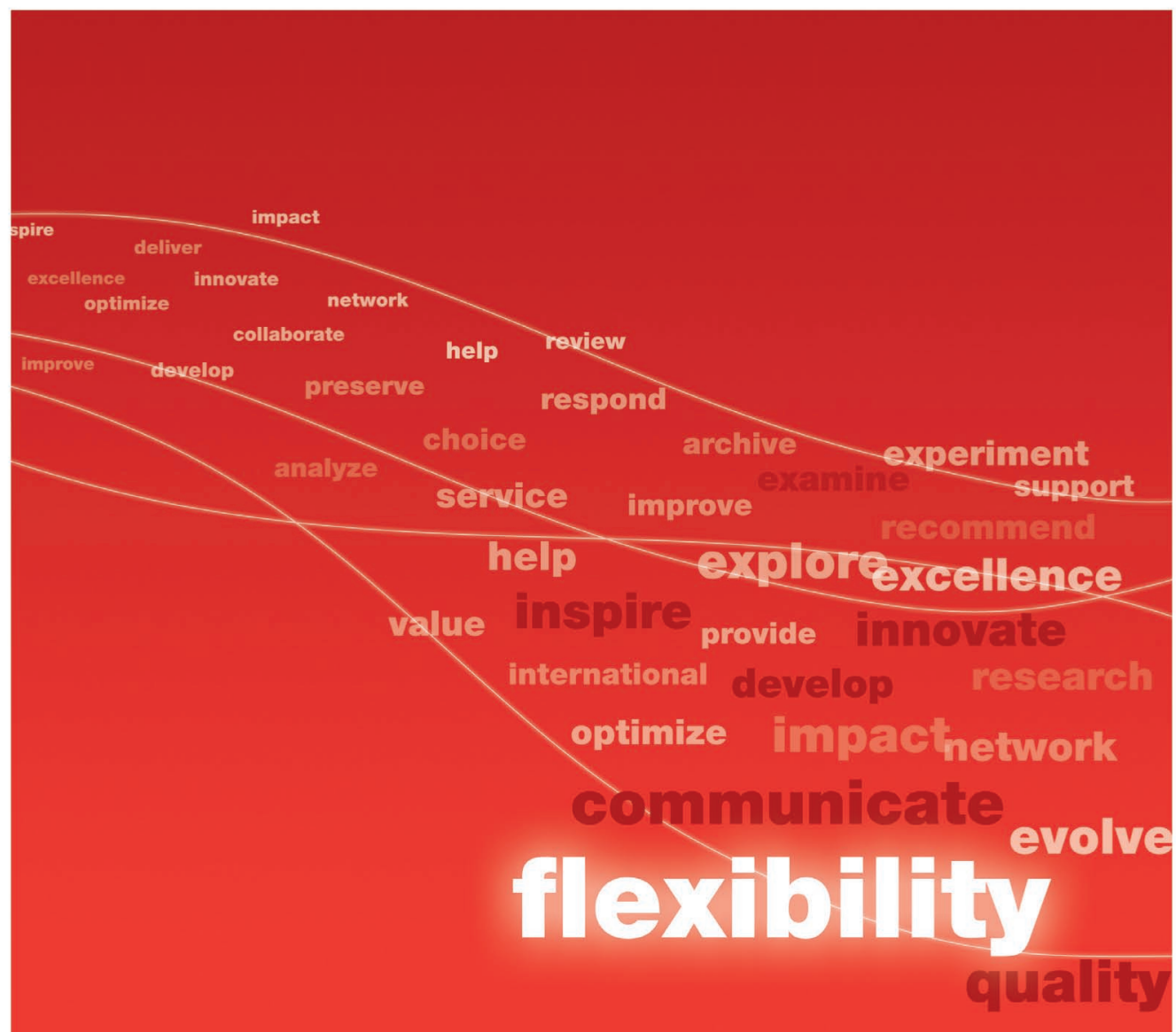

Licencia de acceso es el modelo flexible de NPG

que le permite seleccionar las subscripciones más importantes para su institución. Elige entre publicaciones de alta calidad de Nature Publishing Group y Palgrave Macmillan para crear una licencia que se ajuste a sus requerimientos y a las necesidades de sus usuarios.

Sin paquetes. Total flexibilidad. Su elección.

Para más información, dirijase a www.nature.com/libraries 